\title{
The gut microbiome: a missing link in understanding the gastrointestinal manifestations of COVID-19?
}

\author{
Erin F. Brooks ${ }^{1,2}$ and Ami S. Bhatt ${ }^{1,2}$ \\ ${ }^{1}$ Department of Medicine (Division of Hematology; Division of Blood and Marrow Transplantation), \\ ${ }^{2}$ Department of Genetics, Stanford University, Stanford, California 94305, USA
}

Abstract Coronavirus disease 2019 (COVID-19), which is caused by infection with SARSCoV-2, presents with a broad constellation of both respiratory and nonrespiratory symptoms, although it is primarily considered a respiratory disease. Gastrointestinal symptoms -including nausea, abdominal pain, vomiting, and diarrhea_rank chief among these. When coupled with the presence of viral RNA in fecal samples, the presence of gastrointestinal symptoms raises relevant questions regarding whether SARS-CoV-2 can productively infect the upper or lower gastrointestinal tract. Despite the well-documented prevalence of gastrointestinal symptoms and the high rate of SARS-CoV-2 fecal RNA shedding, the biological, clinical, and epidemiological relevance of these findings is unclear. Furthermore, the isolation of replication-competent virus from fecal samples has not been reproducibly and rigorously demonstrated. Although SARS-CoV-2 shedding likely occurs in a high proportion of patients, gastrointestinal symptoms affect only a subset of individuals. Herein, we summarize what is known about gastrointestinal symptoms and fecal viral shedding in COVID-19, explore the role of the gut microbiome in other respiratory diseases, speculate on the role of the gut microbiota in COVID-19, and discuss potential future directions. Taking these concepts together, we propose that studying gut microbiota perturbations in COVID-19 will enhance our understanding of the symptomology and pathophysiology of this novel devastating disease.

Corresponding author: asbhatt@stanford.edu

(c) 2021 Brooks and Bhatt This article is distributed under the terms of the Creative Commons Attribution-NonCommercial

License, which permits reuse and redistribution, except for commercial purposes, provided that the original author and source are credited.

Published by Cold Spring Harbor Laboratory Press

doi:10.1101/mcs.a006031

\section{GASTROINTESTINAL SYMPTOMS AND FECAL VIRAL SHEDDING IN COVID-19}

Since its zoonotic transfer to humans, SARS-CoV-2 has spread across the globe with unprecedented speed. The associated coronavirus disease 2019 (COVID-19) is widely regarded as a respiratory disease because of the prevalence and severity of symptoms such as cough, sore throat, congestion, anosmia, and dyspnea. Interestingly, gastrointestinal (GI) symptoms, although initially overlooked, are now appreciated as a common manifestation of the disease. In a multicenter cohort study of 318 hospitalized COVID-19 patients in the United States, at admission, 107 (33.7\%) patients reported diarrhea, 84 (26.4\%) reported nausea, $49(15.4 \%)$ reported vomiting, and 46 (14.5\%) reported abdominal pain (Redd et al. 2020). In another cohort study of 254 people in China with COVID-19, 66 (26.0\%) of patients reported at least one of the following Gl symptoms: diarrhea, vomiting, nausea, or abdominal pain (Zhou et al. 2020b). Globally, a meta-analysis of 60 studies that have reported on Gl symptoms on a total of 4243 unique patients found that the pooled prevalence of $\mathrm{Gl}$ symptoms was $17.6 \%$ (95\% confidence interval [Cl], 12.3-24.5) (Cheung et al. 2020). The results of all 
meta-analyses reporting on the prevalence of Gl symptoms on PubMed prior to January 19, 2021 are summarized in Table 1. The true percentage of individuals experiencing Gl symptoms could possibly be far higher given that these symptoms are less likely to merit testing or hospitalization and may be experienced in the absence of recognizable respiratory symptoms (Mao et al. 2020; Xu et al. 2020). In support of this postulated underappreciation of the prevalence of GI symptoms in COVID-19, one retrospective study of hospitalized patients reported that $\mathrm{Gl}$ disturbances were the predominant presenting complaint and initial symptom in 103 (50.5\%) of 204 individuals. Diarrhea was the most common individual Gl symptom experienced by 38 (18.6\%) of patients at admission (Pan et al. 2020), indicating that GI symptoms may not be secondary to respiratory disease, but rather due to a separate biological phenomenon. Perplexingly, the presence of Gl symptoms does not appear to be consistently correlated with respiratory or overall disease severity. In some reports, patients who initially present with Gl symptoms have a greater risk of disease progression, possibly due to delayed diagnosis and treatment (Mao et al. 2020), whereas other preliminary reports have found that $\mathrm{Gl}$ symptoms are associated with milder courses of disease (Livanos et al. 2020).

In addition to Gl symptoms, viral RNA has been detected in stool samples via quantitative real-time polymerase chain reaction (RT-qPCR) assay across multiple studies (Cheung et al. 2020; Parasa et al. 2020; Wang et al. 2020c). In the most recent and most comprehensive meta-analysis of fecal viral shedding to date, including 44 studies of at least 10 patients representing 1989 unique patients, van Doorn et al. (2020) found a pooled prevalence of positive fecal viral tests in $51.8 \%(95 \% \mathrm{Cl}, 43.8-59.7 \%)$ of stool samples or rectal swabs. Table 2 summarizes data on the prevalence and duration of fecal viral shedding compiled by meta-analyses available on PubMed prior to January 19,2021. Other single-cohort studies have found that SARS-CoV-2 genomic viral RNA is shed in feces at viral loads that are comparable to respiratory specimens at their peak (Wölfel et al. 2020); and fecal shedding can persist for weeks after diagnosis, often long after respiratory specimens test negative (van Doorn et al. 2020; Wu et al. 2020). Interestingly, fecal viral shedding has also been reported in asymptomatic individuals, suggesting that a greater number of individuals may be shedding viral RNA in their feces than has been reported (Hua et al. 2020; Park et al. 2020). Although it is tempting to speculate that fecal RNA levels may be associated with the presence or absence of Gl symptoms, to date, there does not appear to be a consistent significant correlation between the presence of fecal viral RNA and GI symptoms across 12 studies that reported on these statistics (van Doorn et al. 2020).

These reports naturally lead to the question of whether SARS-CoV-2 infection of the GI tract drives the underlying etiology of Gl symptoms. Physiologically, there is evidence that this may be possible. Angiotensin-converting enzyme 2 (ACE2), the cellular receptor for SARS-CoV-2, is expressed by nonrespiratory tissues, including the heart and liver, with highest levels present in the small intestine, testes, kidney, and gallbladder (Hamming et al. 2004; Hikmet et al. 2020; Hoffmann et al. 2020; Li et al. 2020). Within the GI tract, ACE2 is expressed at threefold higher levels in the epithelial cells of the upper GI tract (ileum and jejunum) than in the lower Gl tract (colon) (Hikmet et al. 2020) and is coexpressed with transmembrane serine protease 2 (TMPRSS2), a cell surface protein that facilitates viral entry into the cell (Ziegler et al. 2020). This supports the GI tract as a site of SARS-CoV-2 tropism. SARS-CoV-2 has further been shown to productively infect intestinal organoids derived from the upper GI tract (duodenum and ileum) (Lamers et al. 2020; Zang et al. 2020; Zhou et al. 2020a) and preliminary studies report culturing of infectious virus from stool samples of infected individuals (Xiao et al. 2020).

Although COVID-19 is not thought to be a predominantly GI disease and the direct evidence of viral infection of the $\mathrm{Gl}$ tract is limited, framed within a historical context, the idea that SARS-CoV-2 has both $\mathrm{Gl}$ and respiratory tropism is plausible. There is ample evidence of 


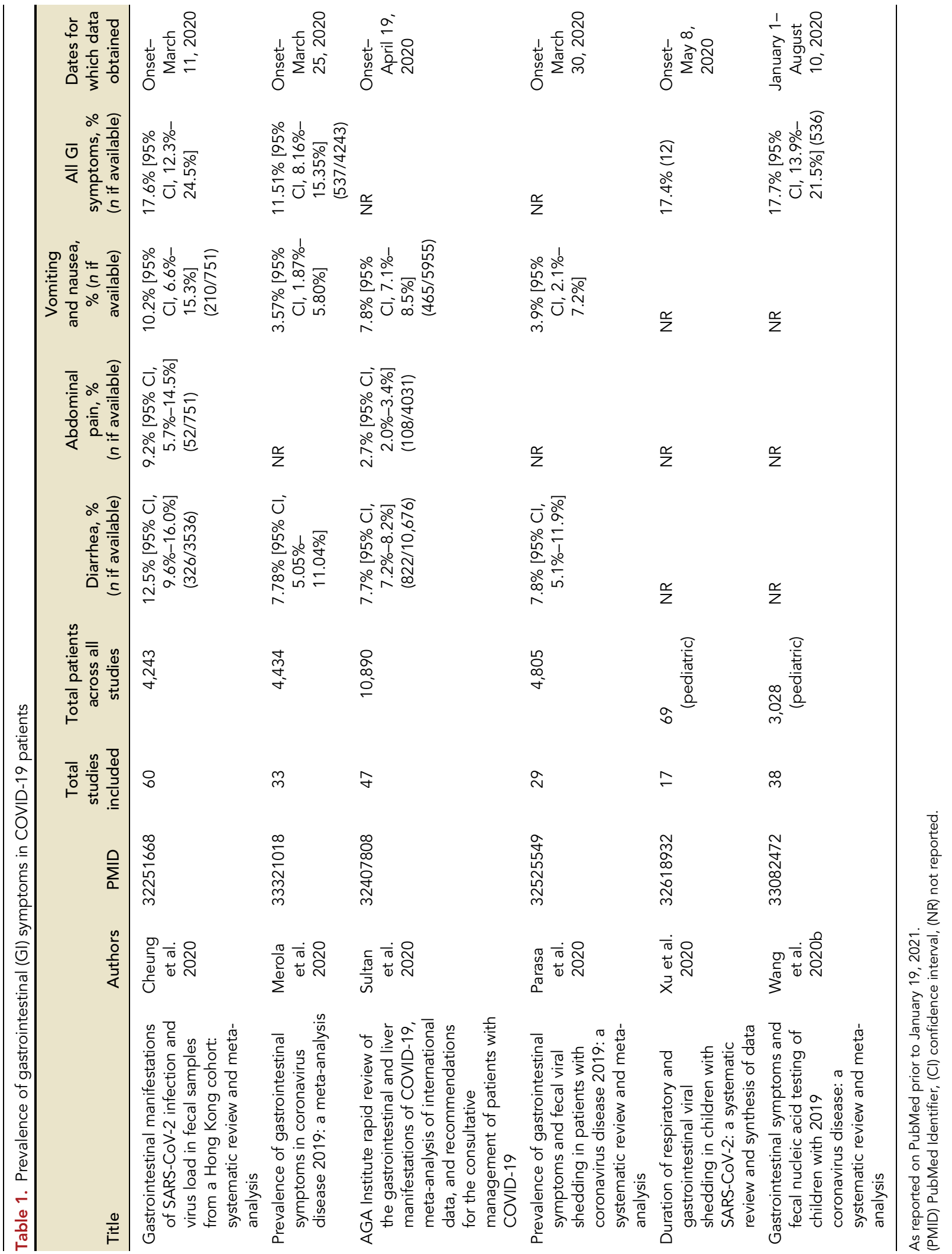




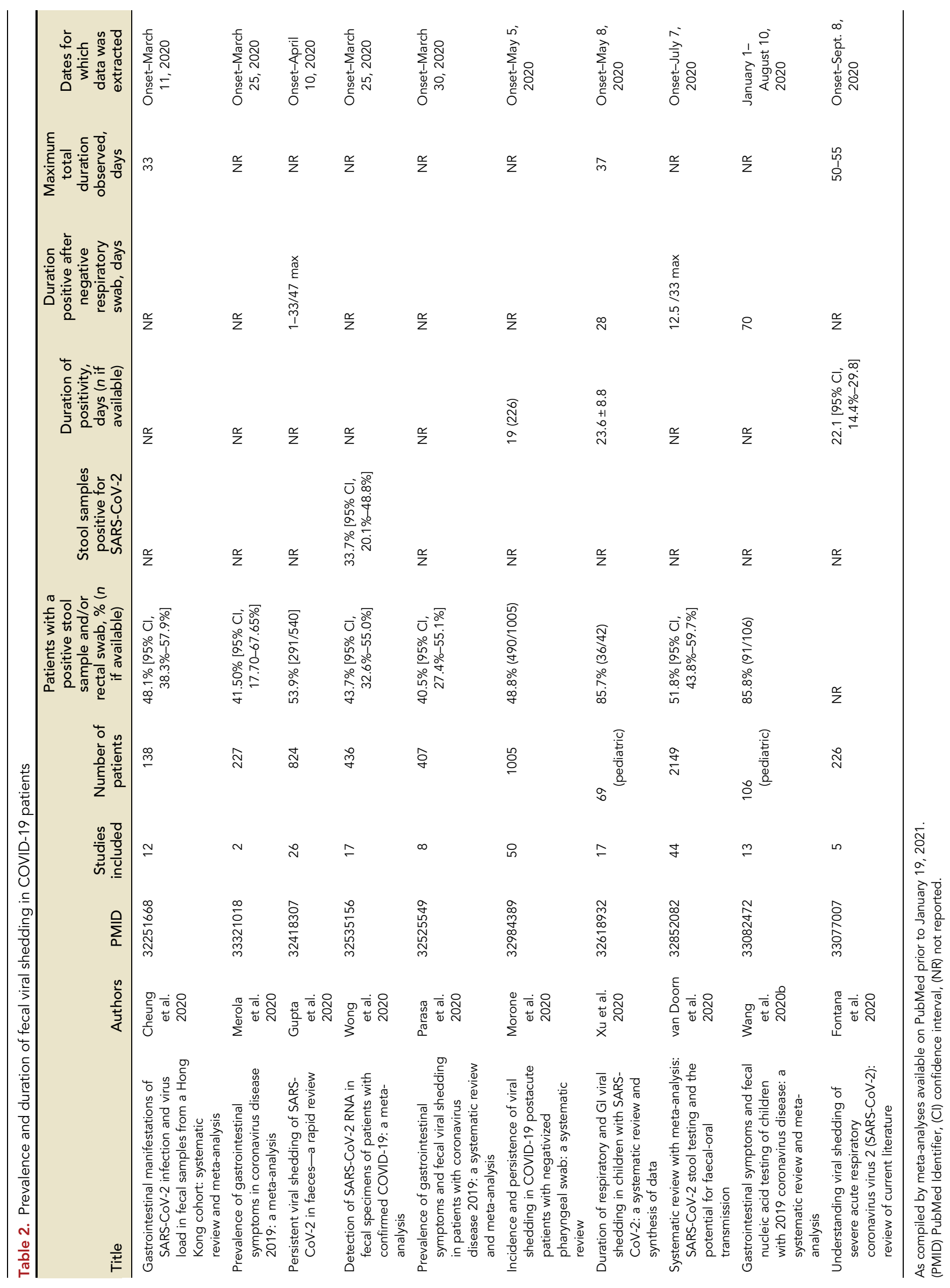


Gl infectivity for the closely related SARS-CoV and MERS-CoV coronaviruses, which were responsible for epidemics in 2003 and 2012, respectively (Peiris et al. 2003b; Zaki et al. 2012). $\mathrm{Gl}$ symptoms, such as diarrhea, were reported for both diseases in about $20 \%$ of SARS-CoV cases and up to $26 \%$ of MERS-CoV cases (Lee et al. 2003; Assiri et al. 2013). Additionally, viral genomic RNA was readily detected in the majority of fecal samples from SARS cases (Peiris et al. 2003a) and roughly one-third of MERS cases (Corman et al. 2016). Live virus could not be propagated from MERS fecal samples. However, SARS-CoV was isolated by culture from tissue biopsies from both the lung and small intestine from patients with SARS (Leung et al. 2003; To et al. 2004). Infection in the epithelial cells of the GI tract of SARS cases was further confirmed by immunofluorescence-fluorescence in situ hybridization (To et al. 2004).

Taken together, this body of evidence suggests SARS-CoV-2 may be capable of causing viral infection in the $\mathrm{Gl}$ tract of affected individuals. However, even in absence of confirmed $\mathrm{Gl}$ infection, the relevance of $\mathrm{Gl}$ symptoms to overall disease severity or duration remains unclear. Several questions remain, particularly the question of which factors contribute to these symptoms and underlie the substantial heterogeneity observed among confirmed COVID-19 cases. One plausible explanation is that SARS-CoV-2 infection leads to perturbation of the gut microbiome toward dysbiosis, which may contribute to the underlying etiology of GI symptoms. Clinically, this may also be a sign of disease progression. SARS-CoV-2 infection is known to occur in two stages, with the initial phase marked by a robust innate immune response and the second phase marked by epithelial barrier injury and inflammation (Tay et al. 2020). Therefore, as a potent modulator of immunity and inflammation, the gut microbiome could potentially serve as a key mediator of COVID-19 symptoms and severity in both stages of disease and a potential target for new therapeutic interventions.

\section{THE ROLE OF THE GUT MICROBIOME IN RESPIRATORY DISEASE}

There is substantial evidence that the gut microbiome plays an influential role in respiratory health and disease. Immunological cross-talk between respiratory and enteric tissues has been well-described and is generally referred to as the gut-lung axis, highlighting the bidirectionality of the relationship. Inflammatory disease in either the lung or Gl tract is sometimes linked to disturbances in the other organ (Budden et al. 2017). For example, chronic obstructive pulmonary disease and inflammatory bowel disease, which are inflammatory diseases affecting the lungs and GI tract, respectively, have both been documented to increase risk of coincident disease within the axis (Keely et al. 2012). It is hypothesized that increased cooccurrence of gut-lung inflammatory diseases may arise from a common mucosal immunological system (Bienenstock et al. 1978). Additionally, commensal gut microbe-derived products and metabolites can induce hematopoiesis, thus providing one mechanism by which gut microbiota may directly influence immune function and regulation in health (Dang and Marsland 2019). The current state of knowledge regarding the relationship between GI microbiota and respiratory tract in the settings of both health and disease are reviewed by Wypych et al. (2019).

In the setting of viral lung infections by noncoronavirus pathogens such as respiratory syncytial virus (RSV) or influenza, certain gut microbes have been implicated as being both altered in response to and protective against infection. In critically ill patients with acute viral pneumonia, both the lung and gut microbiome are significantly altered with a shift toward increased presence of opportunistic pathogens. In the lungs, increased bacterial load, increased alpha diversity, and the presence of gut-associated taxa such as Lachnospiraceae and Enterobacteriaceae are associated with worse prognoses (Kyo et al. 2019; Dickson et al. 2020). Conversely, in the gut, sublethal influenza A H5N1 respiratory infection was shown to deplete the microbiota and diminish community diversity in a mouse model, 
thereby increasing susceptibility to gut invasion by pathogens (Yildiz et al. 2018). In mouse models of RSV and influenza infection, Bacteroidetes, particularly within the Porphyromonadaceae family, increased in relative abundance, whereas organisms in the Lactobacillaceae and Lachnospiraceae families decreased following lung infection but not following vaccination with live attenuated virus (Groves et al. 2018). Other studies in influenza mouse models have shown that type I interferon production in the lungs during respiratory viral infection is one mechanism that can drive gut microbial dysbiosis by depleting obligate anaerobes (Deriu et al. 2016). Some of the observed shifts in gut microbial composition, especially Bacteroidetes outgrowth, may be attributable to decreased dietary caloric intake owing to disease-induced inappetence, as suggested by a study in mouse models (Groves et al. 2020).

Additionally, a growing body of literature has provided mechanistic insight into the various roles that gut commensals play in enhancing innate immunity as well as modulating the balance between type I and type II adaptive immune response (Omenetti and Pizarro 2015). For example, prior to respiratory viral infection, studies in murine models have shown that lipooligosaccharides in the outer membrane of Bacteroides sp., such as polysaccharide A produced by Bacteroides fragilis, prime immune resistance by stimulating interferon- $\beta$ signaling via activation of colonic dendritic cells (Stefan et al. 2020). In the setting of RSV or influenza infection, there is substantial evidence that specific commensal gut taxa, particularly Lactobacillus, can enhance immune resistance (Maeda et al. 2009; Park et al. 2013; Wu et al. 2013; Schuijt et al. 2016; Belkacem et al. 2018; Hanada et al. 2018). Specific microbiota may also play a role in regulating the inflammatory phase, which marks later stages of disease. For example, segmented filamentous bacteria have been shown to promote the development of T-helper 17 cells, thus inducing a pro-inflammatory immune state (Ivanov et al. 2009). Other avenues by which gut microbes prime and modulate immunity and inflammation have been reviewed by Belkaid and Hand (2014). Finally, in mouse models certain gut commensals, like Lactobacillus, boost innate immunity against bacterial respiratory infection. Specifically, these potent NOD-like receptor pattern recognition receptor stimulators enhance alveolar macrophage activity through the GM-CSF signaling pathway (Brown et al. 2017). This suggests that organisms like Lactobacillus may be a therapeutic strategy to prevent against secondary bacterial pneumonia, a common cause of mortality in patients with viral pneumonia. Thus, the existing body of literature provides clear mechanistic evidence that gut commensals are protective and influence prognosis following respiratory infection by pathogens other than SARS-CoV-2. Combined with the knowledge of gut dysbiosis in response to respiratory infection, this lends substantial support to the hypothesis that the gut microbiome may influence the severity of COVID-19 symptoms and outcomes related to the primary infection as well as secondary bacterial pneumonia.

\section{THE ROLE OF THE GUT MICROBIOTA IN COVID-19}

Since the start of the COVID-19 pandemic, many have proposed that the gut microbiome may be an influential factor in COVID-19 pathogenesis, but only a few have provided data in support of this claim. In their preprint, Gou et al. (2020) found correlative evidence to suggest that certain gut microbes may increase risk of more severe COVID-19 in healthy, noninfected individuals. Using a machine learning model, they identified operational taxonomic units in the gut microbiota of 301 healthy participants that explained up to $22 \%$ of the variability in a blood proteomic risk score predictive of COVID-19 disease severity. After testing these in a healthy independent cohort against a panel of pro-inflammatory cytokines, they found significant negative correlations for the Bacteroides and Streptococcus genera and positive associations for the Ruminococcus, Blautia and Lactobacillus genera, thus providing insight into 
a possible mechanism by which certain gut microbes could promote COVID-19 progression after infection. Similarly, in a recent preprint, Ward et al. (2021) applied a random forest machine learning algorithm to identify gut microbial biomarkers of COVID-19 outcomes in a cohort of 62 COVID-19 patients. They found that a combination of features from the gut microbiota were able to predict COVID-19 severity with up to $92.05 \%$ accuracy. Of these, an enrichment of Enterococcus faecalis was one of the top predictors of COVID-19 severity, whereas enrichment for $B$. fragilis was strongly predictive of moderate disease. Together, these preliminary findings suggest that the gut microbiome may have prognostic value in assessing risk of severe COVID-19 outcomes.

Other studies have characterized the composition of the lung or gut microbiota in the setting of SARS-CoV-2 respiratory infection to identify features indicative of disease severity. An analysis of metatranscriptomic sequencing data from bronchoalveolar lavage fluid from eight patients with COVID-19 respiratory disease found similar microbiota compositions to those from patients with community acquired pneumonia-namely, an increase in the number of pathogenic organisms or an increase in commensals normally found in the oral cavity (Shen et al. 2020). A cross-sectional study of the gut microbiota of 30 patients with COVID-19 via 16S ribosomal RNA gene sequencing found significantly decreased Shannon diversity and an increased relative abundance of opportunistic pathogens, including Streptococcus, Rothia, and Actinomyces, as compared to 24 patients with influenza A (H1N1) and 30 healthy matched controls (Gu et al. 2020). Similarly, Zuo et al. (2020a) performed RNA metatranscriptomic sequencing of fecal samples from 15 hospitalized patients with COVID-19 and found a greater predominance of disease-associated microbiota in patients with higher sequencing coverage of the $3^{\prime}$ region of the SARS-CoV-2 viral genome as compared to the $5^{\prime}$ region, potentially indicative of active viral replication in the gut. In seven antibiotic naive patients within this same cohort, baseline gut microbiome shotgun metagenomic sequencing data revealed an enrichment for species including Clostridium hathewayi (reclassified in 2014 to Hungatella hathewayi) (Kaur et al. 2014), Actinomyces viscosus, and Bacteroides nordii and depletion of commensals, such as Faecalibacterium prausnitzii, as compared to six patients with community acquired pneumonia (Zuo et al. 2020b). The small number of subjects analyzed in these studies represents a significant limitation of these findings. However, in a recent study of an expanded patient cohort from the same authors, gut microbiome sampling data and paired blood cytokine profiling of 100 COVID-19 patients was compared with that of 78 healthy participants surveyed before the onset of the pandemic (Yeoh et al. 2021). Although they observed species level differences in the microbiome profile of COVID-19 and non-COVID-19 controls, these were only present at a few percent relative abundance. Interestingly, when they adjusted for antibiotic exposure and age, they found that Faecalibacterium prausnitzii and Bifidobacterium bifidum were negatively correlated with COVID-19 disease severity; F. prausnitzii was also found to be negatively correlated with pro-inflammatory cytokines.

Taken together, these findings begin to paint a picture for the key roles the gut microbiota may play at each stage of disease. First, prior to infection, the preliminary findings by Gu et al. (2020) suggest that microbes of the Ruminococcus, Blautia or Lactobacillus genus might indicate risk of more severe disease. Then immediately following infection, based on studies of RSV and influenza in mouse models, key gut microbes, such as B. fragilis, are capable of activating host antiviral immunity and determining the strength of the initial immune response. In later stages of disease, the loss of commensals such as $F$. prausnitzii and $B$. bifidum and enrichment for pathobionts such as $E$. faecalis may play a role in amplifying destructive inflammatory responses, as suggested by the recent findings from Ward et al. (2021) and Yeoh et al. (2021). Finally, on a community level, the studies by Zuo et al. (2020b) and Gu et al. (2020) indicate that gut dysbiosis is a common outcome following SARS-CoV-2 respiratory infection and is possibly a risk factor for more severe disease. 
In the context of COVID-19, there is also evidence to suggest that the gut microbiome may undergo particular perturbations that are specific to SARS-CoV-2 infection. Besides being the receptor that mediates cellular viral entry, in vitro ACE2 is a chaperone for the neutral amino acid transporter $\mathrm{B}^{\circ} \mathrm{AT} 1$ coexpressed in the small intestinal epithelium (Camargo et al. 2009). As a result, in mouse models, ACE2 deficiency in the GI tract leads to a decrease in neutral amino acid bioavailability and an increase in vulnerability to intestinal inflammation (Hashimoto et al. 2012; Singer et al. 2012; Liu et al. 2015). In particular, insufficient tryptophan bioavailability and the concomitant lack of host- and microbe-derived tryptophan metabolites is linked to defects in expression of a-defensins, a class of antimicrobial peptides that impact gut microbial composition and host-microbial immunological interactions as reviewed by Gao et al. (2018) and He et al. (2018). In this capacity, ACE2 is a key indirect regulator of gut microbial homeostasis. Infection by SARS-COV, which similarly targets the ACE2 receptor for cellular entry, leads to decreased expression of ACE2 (Kuba et al. 2005). Therefore, it is plausible that SARS-CoV-2 mediates deficiency in enteric ACE2, thereby promoting gut microbial dysbiosis, which may drive the underlying etiology of GI symptoms in the setting of COVID-19.

\section{OPEN QUESTIONS}

Overall, the evidence presented here suggests that the Gl system is an important site of disease in a significant proportion of SARS-CoV-2-infected individuals and provides evidence that certain gut microbes may play a role in disease pathogenesis. However, several major questions, which we outline below, remain to be addressed.

Although the finding that SARS-CoV-2 RNA is shed in the feces has clear epidemiological significance, for example, by enabling wastewater surveillance for community spread of disease, the clinical significance has yet to be enumerated. A recent meta-analysis suggests that about $50 \%$ of known COVID-19 positive individuals test positive by fecal viral RNA test (van Doorn et al. 2020). However, there is substantial variability in this number across individual studies, which may in part reflect inconsistencies in the clinical characteristics of the study population (e.g., disease severity, age, comorbidities), stool sampling frequencies, and methods for viral testing. Nevertheless, it is perplexing that only a subset of cases report Gl symptoms or test positive by fecal testing. Moreover, it is unclear what underlying factors explain the observed protracted duration of fecal viral shedding. Across 49 studies, 282 out of $443(64 \%)$ patients shed viral RNA in their stool for an average of $12.5 \mathrm{~d}$ after respiratory specimens tested negative (van Doorn et al. 2020). On one side, this could indicate delayed clearance of the virus from the upper respiratory tract. Alternatively, if viral RNA is shed from the upper or lower GI tract, then prolonged shedding could signal a protracted enteric infection after the resolution of respiratory disease.

An understanding of the underlying etiology of Gl symptoms and fecal viral shedding in COVID-19 will hinge on a detailed analysis of SARS-CoV-2 infection from the perspective of the Gl system. The idea of coronaviruses as respiratory viruses with enteric tropism is not novel. Several coronaviruses productively infect the Gl tract in other animals (e.g., cats, dogs, pigs) and can cause both respiratory and diarrheal disease, as in the case of bovine coronavirus. Furthermore, the presence of Gl symptoms, the weeks-long duration of fecal viral shedding after respiratory disease resolution, and the existence of the SARS-CoV-2 receptor on the intestinal epithelium support the hypothesis that SARS-CoV-2 could be causing protracted GI infection in COVID-19 cases.

In support of this hypothesis, Gaebler et al. (2021) found a more potent and broader range of SARS-CoV-2-neutralizing antibodies in a cohort of 87 COVID-19-convalescent individuals $6 \mathrm{mo}$ after infection as compared to initial levels assessed at $1.3 \mathrm{mo}$ postinfection. 
Continuous antibody evolution, which requires selection for somatic mutants of anti-SARSCoV-2 memory B cells and ongoing antigen exposure in germinal centers, suggests that SARS-CoV-2 antigen may persist in the body long after respiratory disease resolution. In five out of 14 individuals at an average of 4 mo post-COVID-19 infection, intestinal biopsies were found to have detectable SARS-CoV-2 N protein by immunostaining, and in two participants SARS-CoV-2 viral RNA was detected by in situ hybridization. These findings are highly suggestive of protracted SARS-CoV-2 Gl infection in at least a subset of infected individuals, which may explain continued fecal viral detection long after the resolution of respiratory disease. However, there has yet to be conclusive evidence of SARS-CoV-2 Gl infection in humans and the presence of infectious viral particles in fecal samples has not been robustly and reproducibly demonstrated.

Beyond this, the gut and/or lung microbiota may be a key indirect factor capable of protecting against or attenuating respiratory infection and possibly, although unproven, Gl infection. The studies available to date have observed significant perturbations in the microbiota of COVID-19 patients as compared to both healthy controls and hospitalized patients with H1N1 or community acquired pneumonia. However, the underlying cause of this is unclear. SARS-CoV-2 could play an indirect role by inducing gut microbial dysbiosis via the gut-lung axis, or it is possible, although unproven, that it could directly infect the GI tract via ACE2 receptors highly expressed in the small intestine. Regardless of the mechanism, these studies lend support to the hypothesis that GI symptoms could be explained, in part, by gut microbial dysbiosis. Therefore, the gut microbiome may be a heretofore unexplored contributor to COVID-19 disease pathogenesis. If so, this would suggest that the gut microbiome might be an attractive therapeutic target for COVID-19 intervention. Unfortunately, it is difficult to draw concrete conclusions from these studies given that they are heavily confounded by the generally small cohort sizes and the inclusion of only hospitalized patients, who tend to have a greater degree of exposure to antimicrobials. Therefore, a significant remaining question is whether the observed perturbations in the gut microbiome are of prognostic value.

\section{FUTURE DIRECTIONS}

To shed light on these open questions, it is essential that future studies of the gut microbiota in individuals with COVID-19 associate findings with robust and comprehensive metadata in order to ensure that findings can reliably inform clinical decision-making. The gut microbiota of hospitalized patients with moderate to severe COVID-19 can be heavily perturbed by the antimicrobial regimens that tend to be initiated for possible secondary bacterial pneumonia in these settings. In a retrospective study of 138 hospitalized COVID-19 patients, it was reported that $90 \%$ received antiviral therapy and many received antibacterial therapies such as moxifloxacin (64\%), ceftriaxone (24\%), and azithromycin (18\%) (Wang et al. 2020a). Another relevant concern is the prevalence of comorbid Gl diseases that may confound microbiome sampling results in this patient population. However, this may be of lesser concern given that, in one report, out of 103 COVID-19 patients presenting with a GI symptom at hospitalization, only four had underlying comorbid GI disease (Pan et al. 2020). Finally, concomitant infections or superinfections can have a significant impact on gut microbiota composition and must be considered when evaluating microbiome sampling data. Among 140 hospitalized patients with RT-qPCR-confirmed SARS-CoV-2 infection, tests also found the co-occurrence of IgM antibodies for Mycoplasma pneumoniae (five patients), RSV (one patient), and Epstein-Barr virus (one patient). However, there was no radiological evidence of superinfection (Zhang et al. 2020). Capturing robust and comprehensive metadata in future studies will be absolutely essential for determining the degree to which the aforementioned factors confound microbiome sampling data. 
Ideally, future studies that aim to illuminate the role of the gut microbiota in COVID-19 will meet the following criteria. First, they will include a large representative cohort of individuals from the population with mild to moderate disease not requiring hospitalization. Second, they will prospectively sample the gut microbiota longitudinally according to a fixed schedule that includes sampling in the early, late, and postconvalescent stages of disease. Finally, they will capture comprehensive data on all symptoms experienced and medications taken during the entire course of disease. Moreover, analysis of the gut microbiota should be paired with the results of fecal viral testing to inform our understanding of whether viral shedding is linked to gut dysbiosis. These features will be essential qualities of any study that seeks to clearly define the role of the gut microbiome in COVID-19 disease pathogenesis.

Aside from serving as a potential prognostic indicator, the gut microbiota could be therapeutically valuable as a target for new disease interventions. Given the relative dearth of effective interventions, even at this stage in the pandemic, probiotics, prebiotics, and other gut microbiota focused treatments could improve clinical management of disease. A metaanalysis of Gl symptoms reported in 47 studies including 10,890 unique patients found that $\mathrm{Gl}$ symptoms were less prevalent in reports from China $(5.8 \%(95 \% \mathrm{Cl}, 5.3 \%-6.4 \%))$ than in reports from countries outside China (18.3\% (95\% Cl, 16.6\%-20.1\%)) (Sultan et al. 2020). Although speculative, this finding could hint at a possible role for diet in modulating $\mathrm{Gl}$ symptoms. Given the fact that diet is one of the biggest factors shaping gut microbial community composition, dietary interventions are an example of just one avenue to modulate symptoms or severity of disease via the gut microbiome. Another avenue may be through the administration of probiotics. The finding from Yeoh et al. (2021) that Bifidobacterium bifidum, a commonly used probiotic, is associated with decreased disease severity suggests that there may be a role for probiotic agents in modifying patient outcomes. However, this hypothesis remains to be tested in a prospective manner and, until this is done, should be considered speculative. Thus, studies into the gut microbiota may serve as the key to understanding the perplexing heterogeneity in $\mathrm{Gl}$ symptoms and fecal viral shedding in COVID-19, of crucial importance to fundamental knowledge of as well as clinical management of the disease.

\section{ADDITIONAL INFORMATION}

\section{Acknowledgments}

We recognize and wholeheartedly thank Aravind Natarajan, PhD, Soumaya Zlitni, PhD, Summer Vance, BS, and Alvin Han, BS (Stanford Department of Medicine, Genetics) for their illuminating discussion and thoughtful parsing of the literature. We also thank Matthew Grieshop, MS, and Meena Chakraborty, MS, for their careful reading of the manuscript and for providing helpful feedback. Special thanks to Upinder Singh, MD, Pras Jagannathan, MD, and Julie Parsonnet, MD (Stanford Department of Medicine, Infectious Diseases) for contributing their clinical insights on COVID-19. We thank Renu Verma, PhD, and Jason Andrews, MD (Stanford Department of Medicine, Infectious Diseases) and Ben Pinsky, MD, PhD, Malaya Kumar Sahoo, PhD, and Bryan Stevens, MD (Stanford Department of Pathology) for their guidance in interpreting biological data and clinical assays. Finally, we warmly thank Alexandria Boehm, PhD, Nasa SinnottArmstrong, BS, and Marlene Wolfe, PhD (Stanford Department of Civil and Environmental Engineering) for contributing their expert insight into wastewater-based epidemiology.

\section{Referees}

Matthew Kelly

Anonymous
Competing Interest Statement

The authors have declared no competing interest.

Anonymous 


\section{REFERENCES}

Assiri A, Al-Tawfiq JA, Al-Rabeeah AA, Al-Rabiah FA, Al-Hajjar S, Al-Barrak A, Flemban H, Al-Nassir WN, Balkhy $\mathrm{HH}, \mathrm{Al}$-Hakeem RF, et al. 2013. Epidemiological, demographic, and clinical characteristics of 47 cases of Middle East respiratory syndrome coronavirus disease from Saudi Arabia: a descriptive study. Lancet Infect Dis 13: 752-761. doi:10.1016/S1473-3099(13)70204-4

Belkacem N, Bourdet-Sicard R, Taha M-K. 2018. Lactobacillus paracasei feeding improves the control of secondary experimental meningococcal infection in flu-infected mice. BMC Infect Dis 18: 167. doi:10.1186/ s12879-018-3086-9

Belkaid Y, Hand TW. 2014. Role of the microbiota in immunity and inflammation. Cell 157: 121-141. doi:10 .1016/j.cell.2014.03.011

Bienenstock J, McDermott M, Befus D, O'Neill M. 1978. A common mucosal immunologic system involving the bronchus, breast and bowel. Adv Exp Med Biol 107: 53-59. doi:10.1007/978-1-46843369-2_7

Brown RL, Sequeira RP, Clarke TB. 2017. The microbiota protects against respiratory infection via GM-CSF signaling. Nat Commun 8: 1512. doi:10.1038/s41467-017-01803-x

Budden KF, Gellatly SL, Wood DLA, Cooper MA, Morrison M, Hugenholtz P, Hansbro PM. 2017. Emerging pathogenic links between microbiota and the gut-lung axis. Nat Rev Microbiol 15: 55-63. doi:10.1038/ nrmicro.2016.142

Camargo SMR, Singer D, Makrides V, Huggel K, Pos KM, Wagner CA, Kuba K, Danilczyk U, Skovby F, Kleta R, et al. 2009. Tissue-specific amino acid transporter partners ACE2 and collectrin differentially interact with Hartnup mutations. Gastroenterology 136: 872-882.e3. doi:10.1053/j.gastro.2008.10.055

Cheung KS, Hung IFN, Chan PPY, Lung KC, Tso E, Liu R, Ng YY, Chu MY, Chung TWH, Tam AR, et al. 2020. Gastrointestinal manifestations of SARS-CoV-2 infection and virus load in fecal samples from a Hong Kong cohort: systematic review and meta-analysis. Gastroenterology 159: 81-95. doi:10.1053/j gastro.2020.03.065

Corman VM, Albarrak AM, Omrani AS, Albarrak MM, Farah ME, Almasri M, Muth D, Sieberg A, Meyer B, Assiri $A M$, et al. 2016. Viral shedding and antibody response in 37 patients with Middle East respiratory syndrome coronavirus infection. Clin Infect Dis 62: 477-483. doi:10.1093/cid/civ951

Dang AT, Marsland BJ. 2019. Microbes, metabolites, and the gut-lung axis. Mucosal Immunol 12: 843-850. doi:10.1038/s41385-019-0160-6

Deriu E, Boxx GM, He X, Pan C, Benavidez SD, Cen L, Rozengurt N, Shi W, Cheng G. 2016. Influenza virus affects intestinal microbiota and secondary salmonella infection in the gut through type I interferons. PLoS Pathog 12: e1005572. doi:10.1371/journal.ppat.1005572

Dickson RP, Schultz MJ, van der Poll T, Schouten LR, Falkowski NR, Luth JE, Sjoding MW, Brown CA, Chanderraj R, Huffnagle GB, et al. 2020. Lung microbiota predict clinical outcomes in critically III patients. Am J Respir Crit Care Med 201: 555-563. doi:10.1164/rccm.201907-1487OC

Fontana LM, Villamagna AH, Sikka MK, McGregor JC. 2020. Understanding viral shedding of severe acute respiratory coronavirus virus 2 (SARS-CoV-2): review of current literature. Infect Control Hosp Epidemiol 1-10.

Gaebler C, Wang Z, Lorenzi JCC, Muecksch F, Finkin S, Tokuyama M, Cho A, Jankovic M, Schaefer-Babajew D, Oliveira TY, et al. 2021. Evolution of antibody immunity to SARS-CoV-2. Nature doi:10.1038/s41586-02103207-w

Gao J, Xu K, Liu H, Liu G, Bai M, Peng C, Li T, Yin Y. 2018. Impact of the gut microbiota on intestinal immunity mediated by tryptophan metabolism. Front Cell Infect Microbiol 8: 13. doi:10.3389/fcimb .2018 .00013

Gou W, Fu Y, Yue L, Chen G, Cai X, Shuai M, Xu F, Yi X, Chen H, Zhu Y, et al. 2020. Gut microbiota may underlie the predisposition of healthy individuals to COVID-19. medRxiv doi:10.1101/2020.04.22.20076091

Groves HT, Cuthbertson L, James P, Moffatt MF, Cox MJ, Tregoning JS. 2018. Respiratory disease following viral lung infection alters the murine gut microbiota. Front Immunol 9: 182. doi:10.3389/fimmu.2018 .00182

Groves HT, Higham SL, Moffatt MF, Cox MJ, Tregoning JS. 2020. Respiratory viral infection alters the gut microbiota by inducing inappetence. MBio 11: e03236-19. doi:10.1128/mBio.03236-19

Gu S, Chen Y, Wu Z, Chen Y, Gao H, Lv L, Guo F, Zhang X, Luo R, Huang C, et al. 2020. Alterations of the gut microbiota in patients with coronavirus disease 2019 or H1N1 influenza. Clin Infect Dis 71: 2669-2678. doi:10.1093/cid/ciaa709

Gupta S, Parker J, Smits S, Underwood J, Dolwani S. 2020. Persistent viral shedding of SARS-CoV-2 in faecesa rapid review. Colorectal Dis Off J Assoc Coloproctol GB Irel 22: 611-620.

Hamming I, Timens W, Bulthuis MLC, Lely AT, Navis GJ, van Goor H. 2004. Tissue distribution of ACE2 protein, the functional receptor for SARS coronavirus. A first step in understanding SARS pathogenesis. J Pathol 203: 631-637. doi:10.1002/path.1570 
Hanada S, Pirzadeh M, Carver KY, Deng JC. 2018. Respiratory viral infection-induced microbiome alterations and secondary bacterial pneumonia. Front Immunol 9: 2640. doi:10.3389/fimmu.2018.02640

Hashimoto T, Perlot T, Rehman A, Trichereau J, Ishiguro H, Paolino M, Sigl V, Hanada T, Hanada R, Lipinski S, et al. 2012. ACE2 links amino acid malnutrition to microbial ecology and intestinal inflammation. Nature 487: 477-481. doi:10.1038/nature11228

He F, Wu C, Li P, Li N, Zhang D, Zhu Q, Ren W, Peng Y. 2018. Functions and signaling pathways of amino acids in intestinal inflammation. BioMed Res Int 2018: 9171905. doi:10.1155/2018/9171905

Hikmet F, Méar L, Edvinsson Å, Micke P, Uhlén M, Lindskog C. 2020. The protein expression profile of ACE2 in human tissues. Mol Syst Biol 16: e9610. doi:10.15252/msb.20209610

Hoffmann M, Kleine-Weber H, Schroeder S, Krüger N, Herrler T, Erichsen S, Schiergens TS, Herrler G, Wu N-H, Nitsche A, et al. 2020. SARS-CoV-2 cell entry depends on ACE2 and TMPRSS2 and is blocked by a clinically proven protease inhibitor. Cell 181: 271-280.e8. doi:10.1016/j.cell.2020.02.052

Hua C-Z, Miao Z-P, Zheng J-S, Huang Q, Sun Q-F, Lu H-P, Su F-F, Wang W-H, Huang L-P, Chen D-Q, et al. 2020. Epidemiological features and viral shedding in children with SARS-CoV-2 infection. J Med Virol 92: 2804-2812. doi:10.1002/jmv.26180

Ivanov II, Atarashi K, Manel N, Brodie EL, Shima T, Karaoz U, Wei D, Goldfarb KC, Santee CA, Lynch SV, et al. 2009. Induction of intestinal Th17 cells by segmented filamentous bacteria. Cell 139: 485-498. doi:10 .1016/j.cell.2009.09.033

Kaur S, Yawar M, Kumar PA, Suresh K. 2014. Hungatella effluvii gen. nov., sp. nov., an obligately anaerobic bacterium isolated from an effluent treatment plant, and reclassification of Clostridium hathewayi as Hungatella hathewayi gen. nov., comb. nov. Int J Syst Evol Microbiol 64: 710-718. doi:10.1099/ijs.0 .056986-0

Keely S, Talley NJ, Hansbro PM. 2012. Pulmonary-intestinal cross-talk in mucosal inflammatory disease. Mucosal Immunol 5: 7-18. doi:10.1038/mi.2011.55

Kuba K, Imai Y, Rao S, Gao H, Guo F, Guan B, Huan Y, Yang P, Zhang Y, Deng W, et al. 2005. A crucial role of angiotensin converting enzyme 2 (ACE2) in SARS coronavirus-induced lung injury. Nat Med 11: 875-879. doi:10.1038/nm1267

Kyo M, Nishioka K, Nakaya T, Kida Y, Tanabe Y, Ohshimo S, Shime N. 2019. Unique patterns of lower respiratory tract microbiota are associated with inflammation and hospital mortality in acute respiratory distress syndrome. Respir Res 20: 246. doi:10.1186/s12931-019-1203-y

Lamers MM, Beumer J, van der Vaart J, Knoops K, Puschhof J, Breugem TI, Ravelli RBG, van Schayck JP, Mykytyn AZ, Duimel HQ, et al. 2020. SARS-CoV-2 productively infects human gut enterocytes. Science 369: 50-54. doi:10.1126/science.abc1669

Lee N, Hui D, Wu A, Chan P, Cameron P, Joynt GM, Ahuja A, Yung MY, Leung CB, To KF, et al. 2003. A major outbreak of severe acute respiratory syndrome in Hong Kong. N Engl J Med 348: 1986-1994. doi:10.1056/ NEJMoa030685

Leung WK, To K, Chan PKS, Chan HLY, Wu AKL, Lee N, Yuen KY, Sung JJY. 2003. Enteric involvement of severe acute respiratory syndrome-associated coronavirus infection. Gastroenterology 125: 1011-1017. doi:10.1016/j.gastro.2003.08.001

Li M-Y, Li L, Zhang Y, Wang X-S. 2020. Expression of the SARS-CoV-2 cell receptor gene ACE2 in a wide variety of human tissues. Infect Dis Poverty 9: 45. doi:10.1186/s40249-020-00662-x

Liu C, Xiao L, Li F, Zhang H, Li Q, Liu H, Fu S, Li C, Zhang X, Wang J, et al. 2015. Generation of outbred Ace2 knockout mice by RNA transfection of TALENs displaying colitis reminiscent pathophysiology and inflammation. Transgenic Res 24: 433-446. doi:10.1007/s11248-014-9855-3

Livanos AE, Jha D, Cossarini F, Gonzalez-Reiche AS, Tokuyama M, Aydillo T, Parigi TL, Ramos I, Dunleavy K, Lee $B$, et al. 2020. Gastrointestinal involvement attenuates COVID-19 severity and mortality. medRxiv doi:10.1101/2020.09.07.20187666

Maeda N, Nakamura R, Hirose Y, Murosaki S, Yamamoto Y, Kase T, Yoshikai Y. 2009. Oral administration of heat-killed Lactobacillus plantarum L-137 enhances protection against influenza virus infection by stimulation of type I interferon production in mice. Int Immunopharmacol 9: 1122-1125. doi:10.1016/j.intimp .2009 .04 .015

Mao R, Qiu Y, He J-S, Tan J-Y, Li X-H, Liang J, Shen J, Zhu L-R, Chen Y, lacucci M, et al. 2020. Manifestations and prognosis of gastrointestinal and liver involvement in patients with COVID-19: a systematic review and meta-analysis. Lancet Gastroenterol Hepatol 5: 667-678. doi:10.1016/S2468-1253(20)30126-6

Merola E, Armelao F, de Pretis G. 2020. Prevalence of gastrointestinal symptoms in coronavirus disease 2019: a meta-analysis. Acta Gastro-Enterol Belg 83: 603-615.

Morone G, Palomba A, losa M, Caporaso T, De Angelis D, Venturiero V, Savo A, Coiro P, Carbone D, Gimigliano F, et al. 2020. Incidence and persistence of viral shedding in COVID-19 post-acute patients with negativized pharyngeal swab: a systematic review. Front Med 7: 562.

Omenetti S, Pizarro TT. 2015. The Treg/Th17 axis: a dynamic balance regulated by the gut microbiome. Front Immunol 6: 639. doi:10.3389/fimmu.2015.00639 
Pan L, Mu M, Yang P, Sun Y, Wang R, Yan J, Li P, Hu B, Wang J, Hu C, et al. 2020. Clinical characteristics of COVID-19 patients with digestive symptoms in Hubei, China: a descriptive, cross-sectional, multicenter study. Am J Gastroenterol 115: 766-773. doi:10.14309/ajg.0000000000000620

Parasa S, Desai M, Thoguluva Chandrasekar V, Patel HK, Kennedy KF, Roesch T, Spadaccini M, Colombo M, Gabbiadini R, Artifon ELA, et al. 2020. Prevalence of gastrointestinal symptoms and fecal viral shedding in patients with coronavirus disease 2019: a systematic review and meta-analysis. JAMA Netw Open 3: e2011335. doi:10.1001/jamanetworkopen.2020.11335

Park M-K, Ngo V, Kwon Y-M, Lee Y-T, Yoo S, Cho Y-H, Hong S-M, Hwang HS, Ko E-J, Jung Y-J, et al. 2013. Lactobacillus plantarum DK119 as a probiotic confers protection against influenza virus by modulating innate immunity. PLoS One 8: e75368. doi:10.1371/journal.pone.0075368

Park S-K, Lee C-W, Park D-I, Woo H-Y, Cheong HS, Shin HC, Ahn K, Kwon M-J, Joo E-J. 2020. Detection of SARS-CoV-2 in fecal samples from patients with asymptomatic and mild COVID-19 in Korea. Clin Gastroenterol Hepatol Off Clin Pract J Am Gastroenterol Assoc doi:10.1016/j.cgh.2020.06.005

Peiris J, Chu C, Cheng V, Chan K, Hung I, Poon L, Law K, Tang B, Hon T, Chan C, et al. 2003a. Clinical progression and viral load in a community outbreak of coronavirus-associated SARS pneumonia: a prospective study. Lancet Lond Eng/ 361: 1767-1772. doi:10.1016/S0140-6736(03)13412-5

Peiris J, Lai S, Poon L, Guan Y, Yam L, Lim W, Nicholls J, Yee W, Yan W, Cheung M, et al. 2003b. Coronavirus as a possible cause of severe acute respiratory syndrome. Lancet Lond Engl 361: 1319-1325. doi:10.1016/ S0140-6736(03)13077-2

Redd WD, Zhou JC, Hathorn KE, McCarty TR, Bazarbashi AN, Thompson CC, Shen L, Chan WW. 2020. Prevalence and characteristics of gastrointestinal symptoms in patients with severe acute respiratory syndrome coronavirus 2 infection in the United States: a multicenter cohort study. Gastroenterology 159: 765-767.e2. doi:10.1053/j.gastro.2020.04.045

Schuijt TJ, Lankelma JM, Scicluna BP, de Sousa e Melo F, Roelofs JJTH, de Boer JD, Hoogendijk AJ, de Beer R, de Vos A, Belzer C, et al. 2016. The gut microbiota plays a protective role in the host defence against pneumococcal pneumonia. Gut 65: 575-583. doi:10.1136/gutjnl-2015-309728

Shen Z, Xiao Y, Kang L, Ma W, Shi L, Zhang L, Zhou Z, Yang J, Zhong J, Yang D, et al. 2020. Genomic diversity of severe acute respiratory syndrome-coronavirus 2 in patients with coronavirus disease 2019. Clin Infect Dis Off Publ Infect Dis Soc Am 71: 713-720. doi:10.1093/cid/ciaa203

Singer D, Camargo SMR, Ramadan T, Schäfer M, Mariotta L, Herzog B, Huggel K, Wolfer D, Werner S, Penninger JM, et al. 2012. Defective intestinal amino acid absorption in Ace2 null mice. Am J Physiol Gastrointest Liver Physiol 303: G686-G695. doi:10.1152/ajpgi.00140.2012

Stefan KL, Kim MV, Iwasaki A, Kasper DL. 2020. Commensal microbiota modulation of natural resistance to virus infection. Cell 183: 1312-1324.e10. doi:10.1016/j.cell.2020.10.047

Sultan S, Altayar O, Siddique SM, Davitkov P, Feuerstein JD, Lim JK, Falck-Ytter Y, El-Serag HB. 2020. AGA institute rapid review of the gastrointestinal and liver manifestations of COVID-19, meta-analysis of international data, and recommendations for the consultative management of patients with COVID-19. Gastroenterology 159: 320-334. doi:10.1053/j.gastro.2020.05.001

Tay MZ, Poh CM, Rénia L, MacAry PA, Ng LFP. 2020. The trinity of COVID-19: immunity, inflammation and intervention. Nat Rev Immunol 20: 363-374. doi:10.1038/s41577-020-0311-8

To KF, Tong JH, Chan PK, Au FW, Chim SS, Chan KA, Cheung JL, Liu EY, Tse GM, Lo AW, et al. 2004. Tissue and cellular tropism of the coronavirus associated with severe acute respiratory syndrome: an in-situ hybridization study of fatal cases. J Pathol 202: 157-163. doi:10.1002/path.1510

van Doorn AS, Meijer B, Frampton CMA, Barclay ML, de Boer NKH. 2020. Systematic review with meta-analysis: SARS-CoV-2 stool testing and the potential for faecal-oral transmission. Aliment Pharmacol Ther 52: 1276-1288. doi:10.1111/apt.16036

Wang D, Hu B, Hu C, Zhu F, Liu X, Zhang J, Wang B, Xiang H, Cheng Z, Xiong Y, et al. 2020a. Clinical characteristics of 138 hospitalized patients with 2019 novel coronavirus-infected pneumonia in Wuhan, China. J Am Med Assoc 323: 1061-1069. doi:10.1001/jama.2020.1585

Wang J, Cui H, Tang H, Deng X. 2020b. Gastrointestinal symptoms and fecal nucleic acid testing of children with 2019 coronavirus disease: a systematic review and meta-analysis. Sci Rep 10: 17846.

Wang W, Xu Y, Gao R, Lu R, Han K, Wu G, Tan W. 2020c. Detection of SARS-CoV-2 in different types of clinical specimens. J Am Med Assoc 323: 1843-1844. doi:10.1001/jama.2020.3786

Ward DV, Bhattarai S, Rojas-Correa M, Purkayastha A, Holler D, Qu MD, Mitchell WG, Yang J, Fountain S, Zeamer A, et al. 2021. The intestinal and oral microbiomes are robust predictors of COVID-19 severity the main predictor of COVID-19-related fatality. medRxiv doi:10.1101/2021.01.05.20249061

Wölfel R, Corman VM, Guggemos W, Seilmaier M, Zange S, Müller MA, Niemeyer D, Jones TC, Vollmar P, Rothe C, et al. 2020. Virological assessment of hospitalized patients with COVID-2019. Nature 581: 465-469. doi:10.1038/s41586-020-2196-x

Wong MC, Huang J, Lai C, Ng R, Chan FKL, Chan PKS. 2020. Detection of SARS-CoV-2 RNA in fecal specimens of patients with confirmed COVID-19: a meta-analysis. J Infect 81: e31-e38. 
Wu S, Jiang Z-Y, Sun Y-F, Yu B, Chen J, Dai C-Q, Wu X-L, Tang X-L, Chen X-Y. 2013. Microbiota regulates the TLR7 signaling pathway against respiratory tract influenza A virus infection. Curr Microbiol 67: 414-422. doi:10.1007/s00284-013-0380-z

Wu Y, Guo C, Tang L, Hong Z, Zhou J, Dong X, Yin H, Xiao Q, Tang Y, Qu X, et al. 2020. Prolonged presence of SARS-CoV-2 viral RNA in faecal samples. Lancet Gastroenterol Hepatol 5: 434-435. doi:10.1016/S24681253(20)30083-2

Wypych TP, Wickramasinghe LC, Marsland BJ. 2019. The influence of the microbiome on respiratory health. Nat Immunol 20: 1279-1290. doi:10.1038/s41590-019-0451-9

Xiao F, Sun J, Xu Y, Li F, Huang X, Li H, Zhao J, Huang J, Zhao J. 2020. Infectious SARS-CoV-2 in feces of patient with severe COVID-19. Emerg Infect Dis 26: 1920-1922. doi:10.3201/eid2608.200681

Xu Y, Li X, Zhu B, Liang H, Fang C, Gong Y, Guo Q, Sun X, Zhao D, Shen J, et al. 2020. Characteristics of pediatric SARS-CoV-2 infection and potential evidence for persistent fecal viral shedding. Nat Med 26: $502-$ 505. doi:10.1038/s41591-020-0817-4

Yeoh YK, Zuo T, Lui GC-Y, Zhang F, Liu Q, Li AY, Chung AC, Cheung CP, Tso EY, Fung KS, et al. 2021. Gut microbiota composition reflects disease severity and dysfunctional immune responses in patients with COVID-19. Gut doi:10.1136/gutjnl-2020-323020

Yildiz S, Mazel-Sanchez B, Kandasamy M, Manicassamy B, Schmolke M. 2018. Influenza A virus infection impacts systemic microbiota dynamics and causes quantitative enteric dysbiosis. Microbiome 6: 9. doi:10 $.1186 / s 40168-017-0386-z$

Zaki AM, van Boheemen S, Bestebroer TM, Osterhaus ADME, Fouchier RAM. 2012. Isolation of a novel coronavirus from a man with pneumonia in Saudi Arabia. N Engl J Med 367: 1814-1820. doi:10.1056/ NEJMoa1211721

Zang R, Castro MFG, McCune BT, Zeng Q, Rothlauf PW, Sonnek NM, Liu Z, Brulois KF, Wang X, Greenberg HB, et al. 2020. TMPRSS2 and TMPRSS4 promote SARS-CoV-2 infection of human small intestinal enterocytes. Sci Immunol 5. doi:10.1126/sciimmunol.abc3582

Zhang J-J, Dong X, Cao Y-Y, Yuan Y-D, Yang Y-B, Yan Y-Q, Akdis CA, Gao Y-D. 2020. Clinical characteristics of 140 patients infected with SARS-CoV-2 in Wuhan, China. Allergy 75: 1730-1741. doi:10.1111/all.14238

Zhou J, Li C, Liu X, Chiu MC, Zhao X, Wang D, Wei Y, Lee A, Zhang AJ, Chu H, et al. 2020a. Infection of bat and human intestinal organoids by SARS-CoV-2. Nat Med 26: 1077-1083. doi:10.1038/s41591-020-0912-6

Zhou Z, Zhao N, Shu Y, Han S, Chen B, Shu X. 2020b. Effect of gastrointestinal symptoms in patients with COVID-19. Gastroenterology 158: 2294-2297. doi:10.1053/j.gastro.2020.03.020

Ziegler CGK, Allon SJ, Nyquist SK, Mbano IM, Miao VN, Tzouanas CN, Cao Y, Yousif AS, Bals J, Hauser BM et al. 2020. SARS-CoV-2 receptor ACE2 is an interferon-stimulated gene in human airway epithelial cells and is detected in specific cell subsets across tissues. Cell 181: 1016-1035. doi:10.1016/j.cell.2020.04.035

Zuo T, Liu Q, Zhang F, Lui GC-Y, Tso EY, Yeoh YK, Chen Z, Boon SS, Chan FK, Chan PK, et al. 2020a. Depicting SARS-CoV-2 faecal viral activity in association with gut microbiota composition in patients with COVID-19. Gut 70: 276-284.

Zuo T, Zhang F, Lui GCY, Yeoh YK, Li AYL, Zhan H, Wan Y, Chung ACK, Cheung CP, Chen N, et al. 2020b. Alterations in gut microbiota of patients with COVID-19 during time of hospitalization. Gastroenterology 159: 944-955. doi:10.1053/j.gastro.2020.05.048 


\section{COLD SPRING HARBOR Molecular Case Studies}

\section{The gut microbiome: a missing link in understanding the gastrointestinal manifestations of COVID-19?}

Erin F. Brooks and Ami S. Bhatt

Cold Spring Harb Mol Case Stud 2021, 7: a006031 originally published online February 16, 2021 Access the most recent version at doi:10.1101/mcs.a006031

References This article cites 78 articles, 8 of which can be accessed free at: http://molecularcasestudies.cshlp.org/content/7/2/a006031.full.html\#ref-list-1

License This article is distributed under the terms of the Creative Commons Attribution-NonCommercial License, which permits reuse and redistribution, except for commercial purposes, provided that the original author and source are credited.

Email Alerting Receive free email alerts when new articles cite this article - sign up in the box at the Service top right corner of the article or click here. 